\title{
Genome-wide transcriptomic analysis reveals the gene regulatory network that controlled by SRL1 in regulating rice leaf rolling
}

\section{CURRENT STATUS: POSTED}

Research Square

Xizhi Li

GuangXi University

Min Li

Guangxi University

Beibei Zhou

Guangxi University

Jinlin Bao

Guangxi University

Liang Zhu

GuangXi University

Pengbo Xue

Guangxi University

Jun Wang

Guangxi University

Jian Jin

Guangxi University

$\checkmark$ jinjian@gxu.edu.cnCorresponding Author

ORCiD: https://orcid.org/0000-0002-1978-500X

DOI:

$10.21203 / \mathrm{rs} .2 .22853 / \mathrm{v} 1$

\section{SUBJECT AREAS}

Plant Physiology and Morphology Plant Molecular Biology and Genetics

KEYWORDS

SRL1/CLD1, rolling leaf, RNA-seq, bulliform cells, cuticle 
Abstract

Background

SRL1 (SEMI-ROLLED LEAF 1) also named as CLD1 (CURLED LEAF AND DWARF 1), encoding a putative glyphopholipidininol-anchored membrane protein, has been characterized as a gene involved in the regulation of leaf morphology in rice. Mutants of srl1-1 (point mutation) and srl1-2 (transferred DNA insertion) exhibit defects in leaf development resulting in a phenotype with adaxially rolled leaves.

Results

To explore the gene regulatory network of leaf development that controlled by SRL1 in rice, we created a homozygous SRL1 knock out (KO) line by CRISPR/Cas9, which showed defects in leaf development with adaxially rolling. By comparing the leaf transcriptome of a homozygous SRLI KO line (srl1-KO) with the control, a total number of 3,178 genes were identified as differentially expressed genes, of which 1,216 genes were significantly up regulated, while 1,962 genes were down regulated. Further analyses indicated that, a group of known leaf rolling related genes, which involved in bulliform cells and cuticle development such as OsZHD1, OsLBD3-7, RFS, ACL1, CFL1, SND1, OsCESA5 and OsCESA6 were up or down regulated in the srl1-KO.

Conclusions

SRL1 might control leaf rolling by regulating a couple of genes that affecting cytological architecture of leaf cells such as bulliforms and cuticle of leaves.

\section{Full Text}

Due to technical limitations, full-text HTML conversion of this manuscript could not be completed. However, the manuscript can be downloaded and accessed as a PDF.

Figures 
$\mathbf{a}$

SRL1

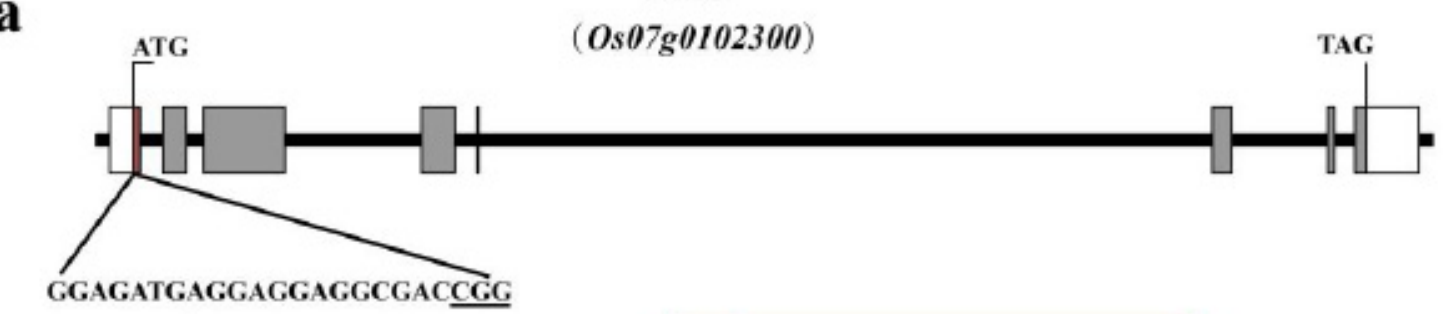

5'-CGCCGCTCGGATGGGGATGGCCCCCGCCGGTCGCCTCCTCCTCATCTCC IGCCTCCTCT-3' 3'-GCGGCGAGCCTACCCCTACCGGGGGC GGC CAGCGGAGGAGGAGTAGAGGACGGAGGAGA-5'

b
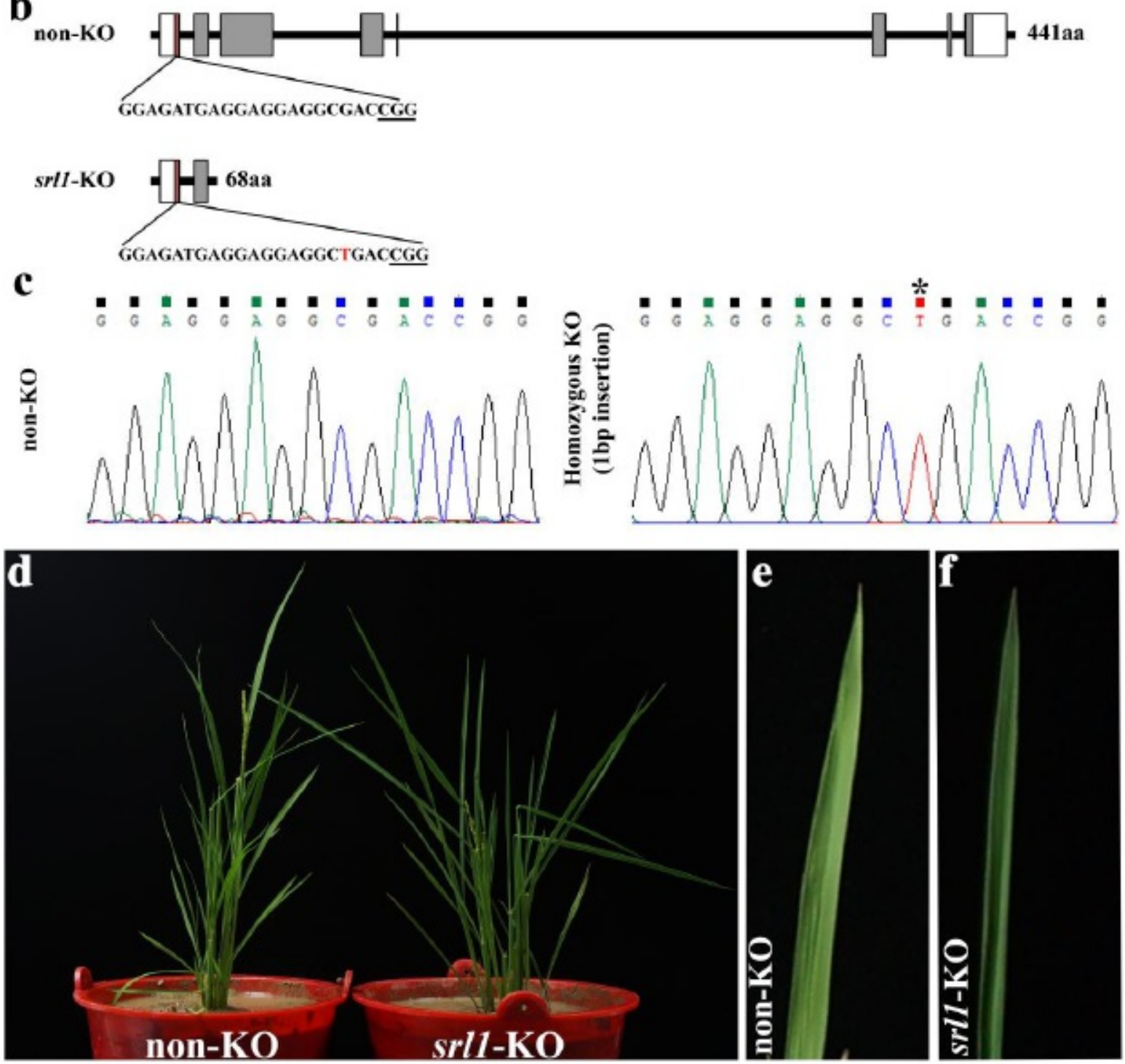

Figure 1

The CRISPR/Cas9 target site of SRL1 and phenotype of the KO plants. (a)Schematic of SRL1 gene structure and the CRISPR/Cas9 target site. The exon was indicated by gray rectangles, 
the untranslated region was indicated by white rectangles. The translation initiation codon (ATG) and termination codon (TGA) are shown. The target site nucleotides are shown in capital letters, and the protospacer adjacent motif (PAM) site is underlined. (b) Genomic structures at the SRLI locus in KO and non-KO line. (c) The original sequencing chromatograms of the target site for SRL1 in non-KO and homozygous KO line. The protospacer adjacent motif (PAM) site is underlined, the " $\mathrm{T}$ " marked with an asterisk was the inserted in the srl1-KO. (d) Whole plant of non-KO and srl1-KO (e, f) Leaf blade phenotype of non-KO and srl1-KO.
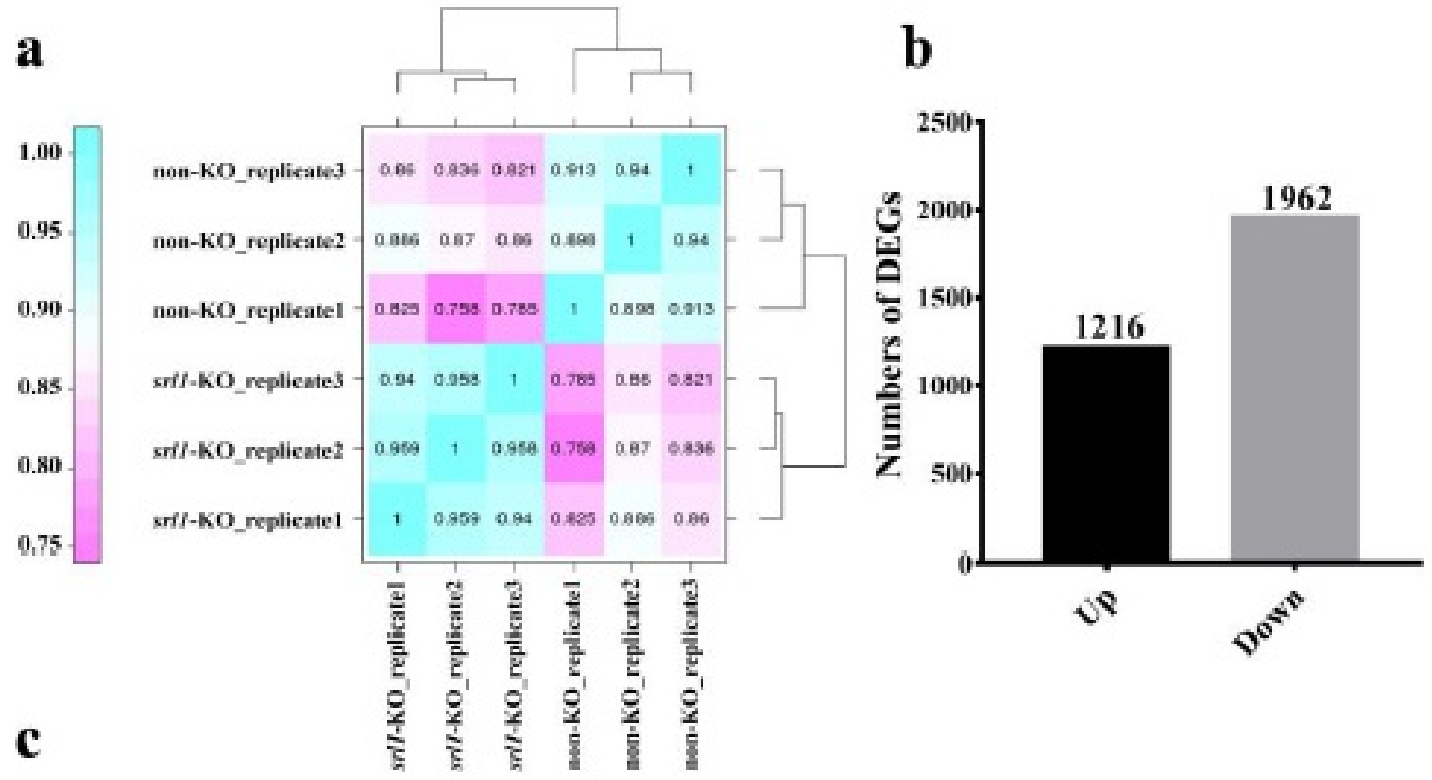

\section{$\mathbf{c}$}
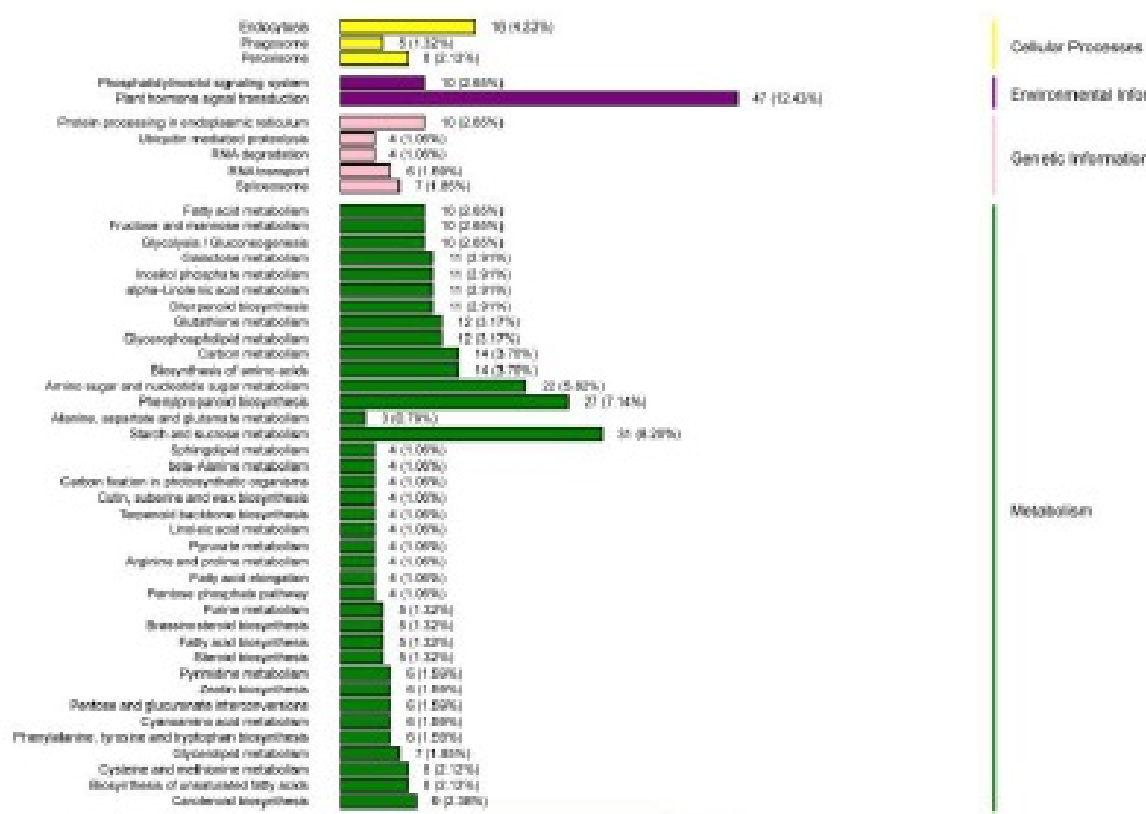

I Erwircenerca hesmeticn Processing

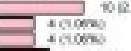

(6in

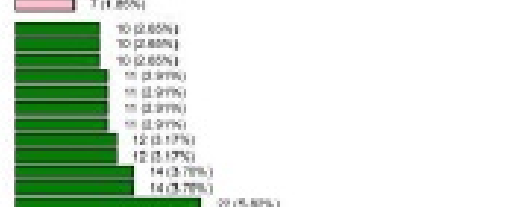

Geietc menneen Processing
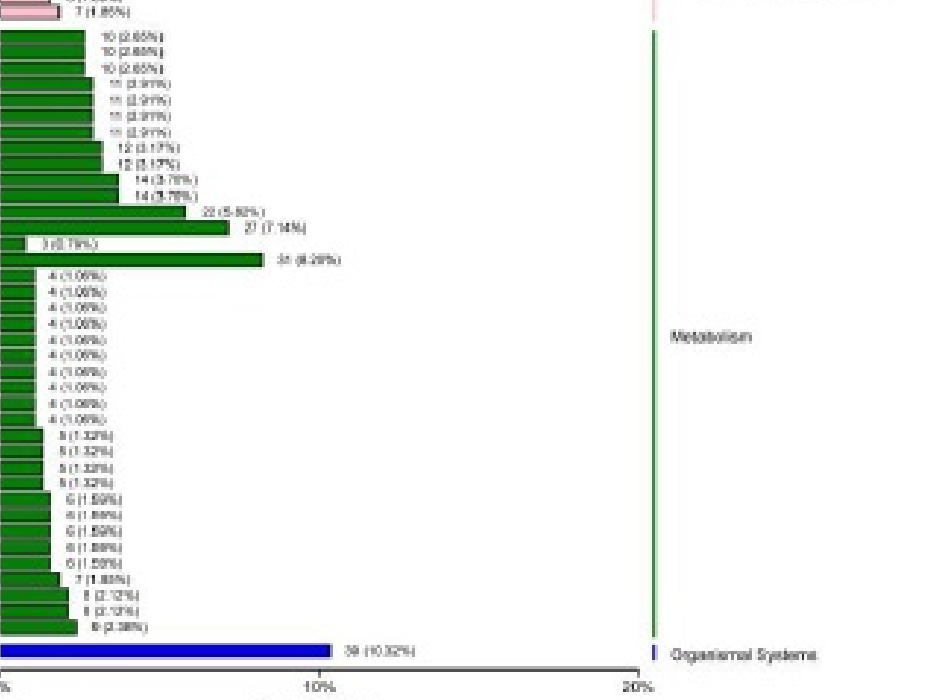
d

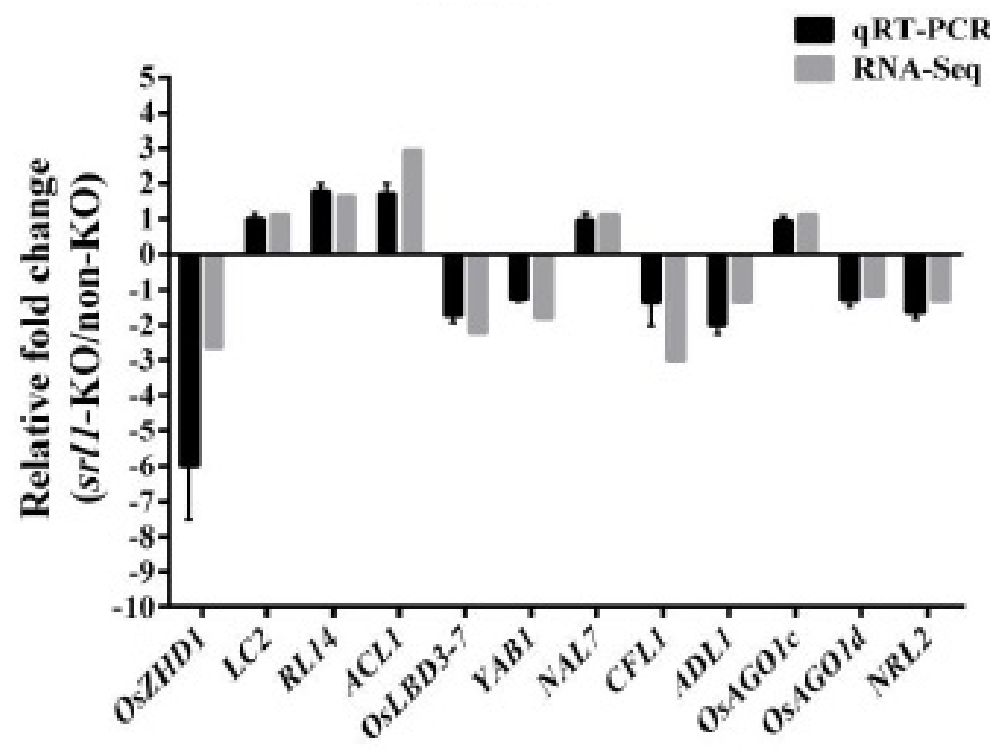

Figure 2

Overview of the DEGs. (a) Correlation between any two replicates was calculated by Pearson's Correlation Coefficient value. (b) Numbers of up-regulated and down-regulated

DEGs. (c) KEGG pathway assignment of the assembled unigenes (top 50 pathways according to enrichment factor). The vertical axis represents the enriched KEGG pathways and the horizontal axis represents the number of unigenes in each pathway. (d) The expression profiles of twelve leaf rolling related genes were validated by qPCR. Values are shown as means \pm s.d. $(n=3)$

\section{Supplementary Files}

This is a list of supplementary files associated with this preprint. Click to download.

Table S5.xIsx

Table S3.xlsx

Table S4.xIsx

Table S1.docx

Table S2.xIsx

Table S6.docx 